\title{
Toward Sheet Testing by Dynamic Bending*
}

\author{
By J. KUČERA,** I. TALPA,** V. $\check{S} M I D^{* *}$ and T. PRNKA ${ }^{* *}$
}

\section{Synopsis}

Behaviour of specimens arranged of steel sheets of various thickness into a V-Charpy sample under conditions of dynamic bending has been investigated. The theoretical analysis into the dependence of the sheet thickness $B_{1}$ on the "crack driving force" $G_{c}$ shows that the function $G_{c}\left(B_{1}\right)$ can be split into four zones characterized by different course of fracture. In the first zone ductile cleavage accurs and the $G_{c}\left(B_{1}\right)$ relation is linear. The second zone is characterized by origination of complex configuration of the fracture surfaces of comparable thickness, which results to fractures welldemanding from the view-point of energy. In the third zone the mixed fracture originates. That part of curve $G_{c}\left(B_{1}\right)$ is originating in principle by the transient curves $G_{c}(t)$ of specimens of a greater thickness. The fourth zone is characterized by occurrence of brittle fractures only.

From the experimental part of the work it followes, that the results acquired with the structural weldable steels are in reasonable conformity with the following equation

$Z \cdot B_{1}^{k_{5}}=k_{6}$
where, $Z=\frac{\mathrm{KGV}_{\max }-\mathrm{KGV}_{\min }}{R_{p \text { dyn }} B_{1}} \frac{|t p|}{t}$

is a dimensionless parameter describing behaviour of specimens at the transition from a cleavage to ductile fracture.

\section{Introduction}

At the acceptance of material in the metallurgical plants the resistance to brittle and ductile fracture is commonly determined by the Charpy $\mathrm{V}$ test. According to the relevant standards the least thickness of specimens is $5 \mathrm{~mm}$. The resistance of steel sheets with a thickness less than $5 \mathrm{~mm}$ is examined under laboratory conditions by a more labour-consuming method based on the fracture mechanics. A steel sheet with crack is loaded by forces acting perpendicularly to the front surfaces of the crack and the conditions of crack propagation are investigated. The testing method of sheets by dynamic bending on specimens of Charpy V shape composed of a pack of sheets was described in Ref. 1). The number of sheets joined in the pack by means of two rivets varied from two to eight.

Reference 1) showed reasonable resolution power of that method for the sheets made of various steel grades. The main advantage of that testing procedure consists in easy preparation of specimens and in possible utilization of common testing equipment available in any mechanical testing laboratory. Because of these advantages, we have applied that method at our study into the behaviour of thin specimens aimed at verification of its resolution power and applicability at material acceptance.

In the course of material acceptance the tests can be carried out either at the ambient temperature or at lower temperatures. In either case the fracture behaviour of the individual sheets in a pack varies either in the field of the ductile fracture or in its neighbourhood because the transient temperatures are decreasing with the thickness of the parts of a specimen. Accordingly, let us deal above all with the well-known facts about the fractures.

\section{Shear and Mixed Fractures}

To appreciate the force conditions in the vicinity of a crack with the help of the fracture mechanics, the parameter $G_{c}$ called "crack driving force" is widely used and by its dimension it is force per unit length. Its course in dependence on the specimen thickness is schematically illustrated in Fig. 1.

It is obvious that this course will be qualitatively the same for both the slow phenomena such as the static measurement of the fracture toughness and the quick phenomena such as the dynamic bending tests of Charpy type and/or the dynamic-tear tests (D-T tests). At dynamic testing the specific energy $\mathrm{KGV}$ consumed per unit area of fracture is rather applied than $G_{c}$; the former has equivalent dimension to the latter $\left(G_{c}\right)$. Let us follow now three fields of thickness of $B$-pieces in which the shear or mixed fracture is originating (see Fig. 1). The fourth field representing purely brittle fractures will not be discussed here.

For the fields 1 and 2 the literature ${ }^{2-4)}$ presents, for the energy consumed for fracture, the following equation

$$
U=C \cdot B^{n}(w-a)^{m}
$$

where, $C, n, m: \quad$ material constants

$B$ : the thickness of specimen

$w$ : the width of specimen

$a$ : the initial length of notch.

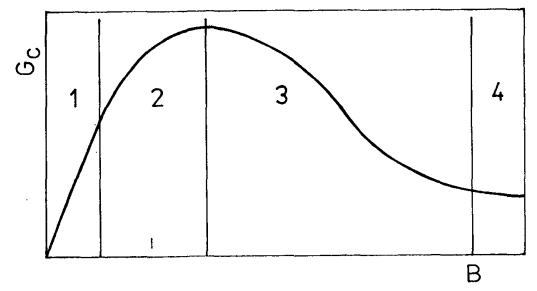

Fig. 1. Gurve showing the dependence of the crack driving force on the thickness of a part. 
For very thin specimens of field 1 (Fig. 1) there are given $n=2$ and $m=1$. Here, the constant $C$ is called the shear fracture energy per unit volume and in literature 4) it is denoted $\varphi$. Thus, Eq. (1) can be rewritten

$$
\frac{U}{B(w-a)}=\mathrm{KGV}=\varphi B
$$

On the basis of application of the theory of dislocations to describe the plastic zone at the front of a crack, the following formula

$$
G_{c}=\frac{2 \sqrt{2} R_{e \mathrm{~L}} B}{(1+\mu)}=2 R_{e \mathrm{~L}} B
$$

is applicable at slow (static) testing of a steel sheet according to the 3rd mode of stress ${ }^{5}$; in this expression $R_{e \mathrm{~L}}$ means lower yield point. At application of the above-cited dimensional equivalence of the crack driving force $\left(G_{c}\right)$ with specific fracture energy $\mathrm{KCV}$ it can be written

$$
\mathrm{KGV}=2 R_{p \mathrm{dyn}} B
$$

Here, the dynamic yield limit $R_{p \text { dyn }}$ takes into account the fact that generation and motion of dislocations at higher strain rates encounter at higher stresses. Equations (3) and (4) define mathematically the first field of the diagram shown in Fig. 1 for the case of slow and quick loading.

In the available literature the field 2 is not considered and the authors lay a boundary between the field 1 and the field 3 (Fig. 1) up to the maximum of the dependence $G_{c}(B)$. However, it can be shown that the field 1 is penetrating into thicknesses of the order of $1 \mathrm{~mm}$, where the maximum is by far not reached. A complex interaction of the planes of maximum shear stress at the surface and inside the specimen takes place in the field 2. An informative review of the differences in the static state of stress is given in Fig. 2 in which the shear lines are drawn for a flat rod subjected to loading by a bending moment $M .^{5)}$

The crack in sample shown in Fig. 2(a) will propagate in a plane inclined by 45 deg towards the sketch plane with path in the line segment $A B$. In the middle of a thicker specimen the planes of the maximum shear stress are perpendicular to the sketch plane with paths lying in the slip lines. The fracture is passing along the arcs $A B$ (Fig. 2(b)). Arrange-

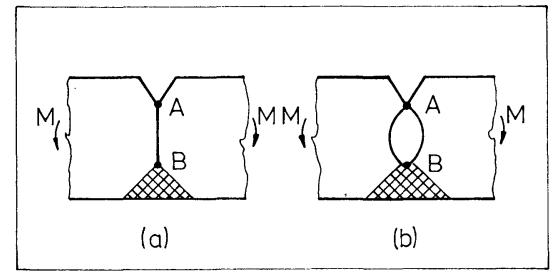

(a) Plane stress condition

(b) Plane strain condition

Fig. 2. Illustration of the shearing lines at bending a flat rod. ment of the two systems in material layers of comparable thickness in a sample leads to more-demanding cracks from the viewpoint of energy if compared with the condition when one of these systems is prevailing (field 1 or 3 ).

In the case of specimen subjected to dynamic stress it is hardly possible to appreciate, whether and in which extent the schemes of Fig. 2 could be applied. This depends on the time of duration of the phenomenon, the absolute size of specimen, the rate of propagation of the elastic waves in material etc. For this reason let us assume the ratio of the individual components of the stress tensor $\sigma_{1}, \sigma_{2}, \sigma_{3}$ (Fig. 3(a)) to the yield limit to be kept-on constant as in the case of a static example. Then, the Mohr's circles for such a specimen thickness can be drawn so as the stress $\sigma_{3}$ would reach $\left(\sigma_{1}+\sigma_{2}\right) \mu$ in the centre, where $\mu$ is the Poisson number. The stresses for plane strain condition in the middle are $\sigma_{1} \sim 2.5 R_{e \mathrm{~L}}, \sigma_{2} \sim 1.5 R_{e \mathrm{~L}}$ and $\sigma_{3} \sim 2 R_{e \mathrm{~L}}$. Here, the magnitude of $\sigma_{1}$ is approaching the fracture stress (the nominal stress at fracture of a tensile test $R_{f}=R_{p} /(1-\psi)$, where $R_{p}$ is the conventional stress at fracture and $\phi$ is the transverse reduction in area of material). The increase in stress rate causes the dynamic yield limit to increase, while the fracture stress $R_{f}$ is rising negligibly. ${ }^{6)}$ The Mohr's circles are displacing to the righthand side (Fig. 3(c)) till the stress $\sigma_{1}$ attains $R_{f}$ and till a crack is initiating in the specimen due to material decohesion. However, the fracture conditions are not still reached at the surface. Further energy should be supplied to reach the shear-fracture stress of $\sigma_{1} / 2=\tau_{f}=R_{f} / 2$ (Fig. 3(b)). Then, the stiffening effect of the marginal shear lips is finished and the entire phenomenon can be repeated for further increment in the length of crack.

Till now no mathematical formulation of field 2 as shown in Fig. 1, similar to Eqs. (3) and (4), has been elaborated. For the shear fractures of the D-T tests belonging here, Eq. (1) can be used with the coefficients $n=0.5$ and $m=2$ for a stee ${ }^{2)}$ and/or $n=$ 0.7 and $m=1.8$ for Al-alloys. ${ }^{3)}$ Here, the constant $C$ has the dimension quite different from $\varphi$ and is called the plastic instability resistance factor. It is possible to recalculate the fracture energy from the Charpy $\mathrm{V}$ tests to the D-T tests on the basis of the volume

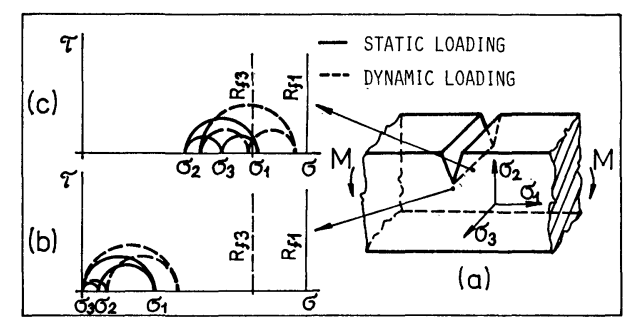

(a) Scheme of loading the specimen

(b) The Mohr's circles of the state of stress for the surface of specimen

(c) The Mohr's circles of the state of stress for the centre of specimen

Fig. 3. Analysis of the state of stress at bending a flat rod. 
shearing energy of fracture $\varphi$ as published elsewhere. ${ }^{4)}$ In view of Refs. 7) and 8) and on the basis of the results obtained in this work it is possible to consider this recalculation to be free of any physical basis as the volume shearing energy $\varphi$ is constant only in the field 1 (Fig. 1) with too low thicknesses.

The field of mixed fractures in Fig. 1 is denoted by 3. The fracture is here partially ductile and partially brittle. It can be mathematically expressed by the following equation ${ }^{4}$

$$
G_{\mathrm{Ic}}=\frac{B_{s}^{2}}{B} \varphi+\frac{B-B_{s}}{B} G_{\mathrm{Ic}}
$$

where, $B_{s}:$ the total width of the shear lips

$G_{\text {Ic }}$ : the fracture toughness of material for a given temperature.

Equation (5) is valid for $B \geq B_{s}$. In the case of $B \gg$ $B_{s}$ the first term at the right-hand side of Eq. (5) is negligible and thus, $G=G_{\text {Ic }}$.

The thickness limiting the field 3 in Fig. 1 is expressed by the following condition

$$
B \geq 2.5\left(\frac{K_{\mathrm{Ic}}}{R_{e \mathrm{~L}}}\right)^{2}=\frac{2.5 G_{\mathrm{Ic}} E}{\left(1-\mu^{2}\right) R_{e \mathrm{~L}}^{2}}
$$

where, $E$ : the Young's modulus.

The diagram in Fig. 1 shows the driving forces of a crack in dependence on the thickness of specimen for a single temperature. At another temperature the course will be quite different. To get an idea about the mutual effect of thickness and temperature Fig. 4 shows schematically the dependence of $G_{c}(B, t)$ with the hatched areas $G_{c}(B)$ and $G_{c}(t)$. It is obvious that the zone of mixed fractures 3 (see Fig. 1) is given by the transient curves of pieces with a greater thick-

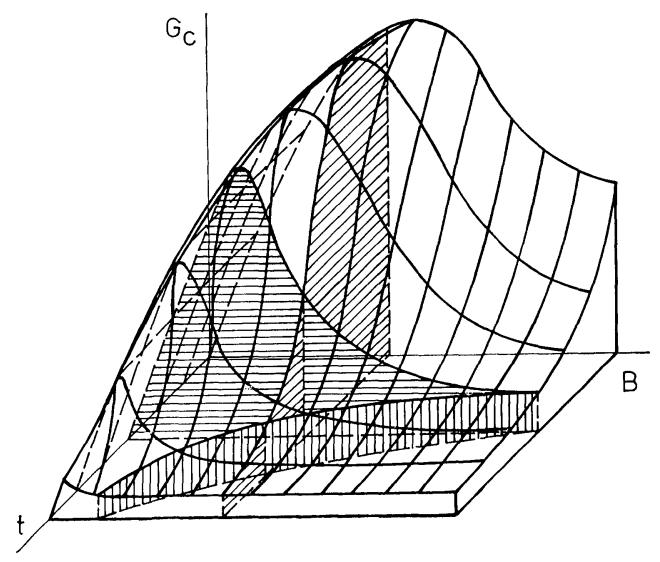

Fig. 4. Dependence of the effect of temperature and thickness of material on the crack driving force. ness, $B$. With increasing temperature the maximum $G_{c \text { max }}$ is being displaced towards greater thicknesses. That displacement has been experimentally studied ${ }^{7,8)}$ on the D-T tests and the following empirical relationship was derived

$$
G_{c \max }=k_{1} \ln B-k_{2}
$$

where, $k_{1}, k_{2}$ : material constants.

In this equation the effect of temperature is implicitly involved as each couple of $G_{c \text { max }}, B$ refers to another temperature as is obvious from Fig. 4.

Moreover, there is obvious the idea about the threshold temperature $t_{p}$ governing the value of $G_{c P}$ at the threshold of dependence $G_{c}(t)$. As given in Ref. 8), $G_{c P}$ is slightly increasing with the thickness and it can be expressed by a relationship analogous to Eq. (7)

$$
G_{c \mathrm{P}}=k_{3} \cdot \ln B-k_{4}
$$

where, $k_{3}, k_{4}$ : again material constants.

This almost constant value owing to the thickness of sample is however achieved at various couples of the variables $B$ and $t_{p}$ as shown schematically in Fig. 4 by a vertically hatched area.

\section{Applied Material and Experimental}

The specimens have been prepared of steel sheets $30 \mathrm{~mm}$ thick of three different materials denoted A, $\mathrm{B}$ and $\mathrm{C}$. The chemical composition of the individual sheet is given in Table 1. Sampling of material A was made of two sites of the very sheet, henceforward denoted A1 and A2. In any case the samples were taken in longitudinal direction, i.e., the crack has propagated perpendicularly to the as-rolled grains.

The relevant parts of specimens 1, 2, 2.5 and $5 \mathrm{~mm}$ thick have been taken par hazard owing to the sheet thickness. For the sake of comparison the checking Charpy V samples $10 \mathrm{~mm}$ thick were prepared. Photograph 1 shows some specimens prior to and after fracture $\left(-10^{\circ} \mathrm{G}\right)$. The surface of all the relevant parts of the composed specimens has been longitudinally ground and the resultant surface unevenness was $4 \times 10^{-6} \mathrm{~m}$ max.

To check the surface effect some samples were prepared of Al-material composed of five parts, milled transversally (the tool traces in parallel with crack propagation) at which the surface unevenness was 16 and $32 \times 10^{-6} \mathrm{~m}$.

The mechanical properties of the examined sheets, determined by the simple tensile test at the ambient

\begin{tabular}{|c|c|c|c|c|c|c|c|c|c|c|c|c|c|}
\hline \multirow{2}{*}{ Material } & \multicolumn{13}{|c|}{ Chemical composition (wt \%) } \\
\hline & G & $\mathrm{Mn}$ & $\mathrm{Si}$ & $\mathrm{P}$ & $\mathrm{S}$ & $\mathrm{Gr}$ & $\mathrm{Ni}$ & Mo & V & $\mathrm{Ti}$ & $\mathrm{Cu}$ & $\mathrm{Al}$ & $\mathrm{Nb}$ \\
\hline A & 0.22 & 1.34 & 0.46 & 0.020 & 0.023 & 0.057 & 0.038 & 0.03 & 0.002 & 0.002 & & & \\
\hline B & 0.08 & 0.44 & 0.22 & 0.011 & 0.014 & 0.04 & 0.06 & & & & 0.04 & 0.037 & \\
\hline C & 0.17 & 1.30 & 0.30 & 0.014 & 0.017 & 0.06 & 0.04 & & & & 0.05 & 0.020 & 0.03 \\
\hline
\end{tabular}
temperature, are listed in Table 2. Here $R_{e \mathrm{~L}}$ means the lower yield point, $R_{m}$ the tensile strength and $R_{f}$

Table 1. The chemical composition of the examined materials. 
Table 2. The mechanical properties of the examined materials.

\begin{tabular}{|c|c|c|c|c|c|c|c|c|}
\hline \multirow{3}{*}{ Material } & \multicolumn{8}{|c|}{ Direction of sampling } \\
\hline & \multicolumn{4}{|c|}{ Longitudinal } & \multicolumn{4}{|c|}{ Transversal } \\
\hline & $\begin{array}{c}R_{e \mathrm{~L}} \\
(\mathrm{MPa})\end{array}$ & $\begin{array}{c}R_{m} \\
(\mathrm{MPa})\end{array}$ & $\begin{array}{l}R_{p \text { dyn }} \\
(\mathrm{MPa})\end{array}$ & $\begin{array}{c}R_{f 1} \\
(\mathrm{MPa})\end{array}$ & $\begin{array}{c}R_{e \mathrm{~L}} \\
(\mathrm{MPa})\end{array}$ & $\begin{array}{c}R_{m} \\
(\mathrm{MPa})\end{array}$ & $\begin{array}{l}R_{p \text { dyn }} \\
(\mathrm{MPa})\end{array}$ & $\begin{array}{c}R_{f 3} \\
(\mathrm{MPa})\end{array}$ \\
\hline A & 410 & 640 & 700 & 1210 & 410 & 640 & 700 & $<930$ \\
\hline B & 210 & 371 & 550 & 931 & 210 & 371 & 550 & 931 \\
\hline G & 343 & 508 & 680 & 1178 & 343 & 508 & 680 & $<1019$ \\
\hline
\end{tabular}

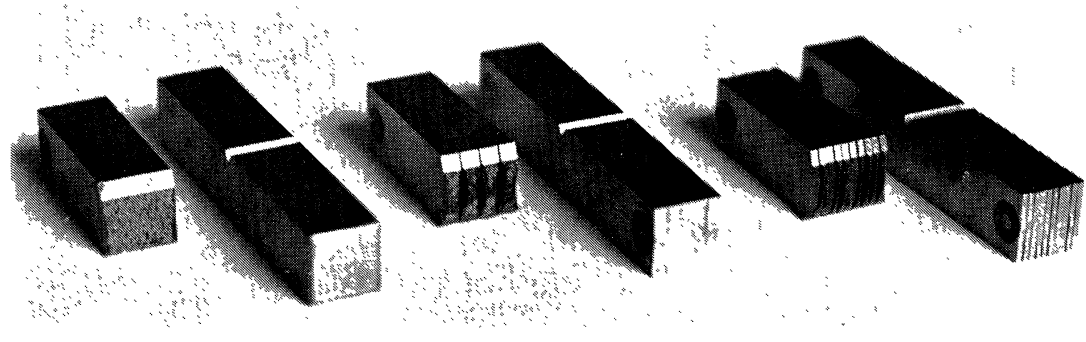

Photo. 1. Appearance of the tested specimens.

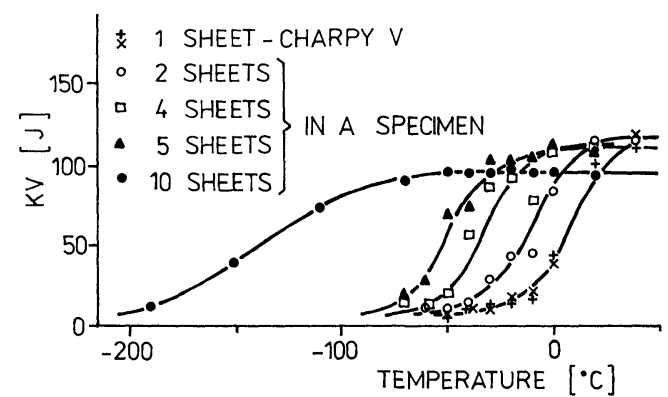

Fig. 5. Gurves showing the dependence of the notch toughness on temperature for material Al.

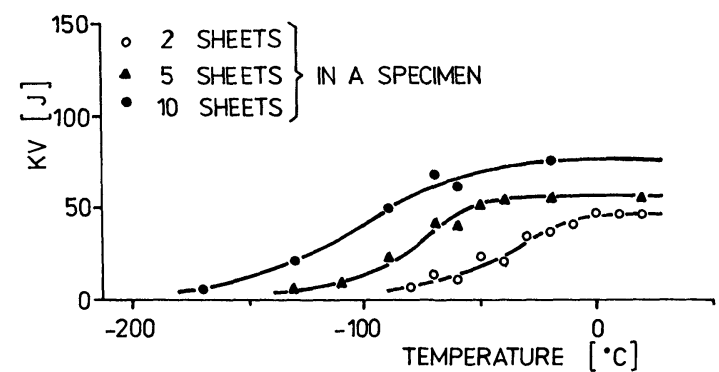

Fig. 6. Curves showing the dependence of the notch toughness on temperature for material A2.

the fracture stress of the tensile specimen. Moreover the dynamic yield point $R_{p \text { dyn }}$ determined approximately for velocity of deformation $\dot{\varepsilon} \sim 4.10^{2} \mathrm{~s}^{-1}$ is added for each grade of steel.

The proper tests have been performed with a pendulum impact machines of Charpy $300 \mathrm{~J}$ type, whereby one of the machines was equipped with electronic instruments to measure the course of force in relation with time. The specimens were cooled down either by nitrogen vapour in a temperature chamber or in the mixture of ethanol with dry ice.

The results of measurements are plotted in Figs. 5 to 9 . Each point is the average of three to four measurements. There is shown the course of the consumed energy in relation with temperature and for

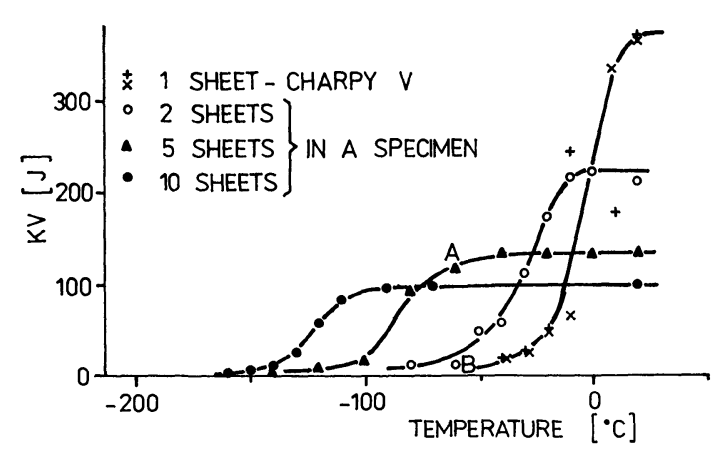

Fig. 7. Curves showing the dependence of the notch toughness on temperature for material B.

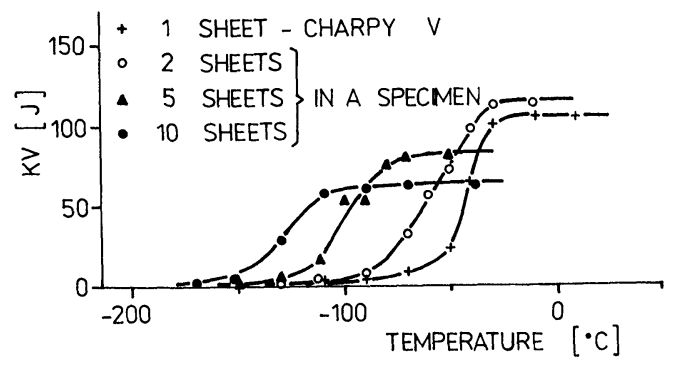

Fig. 8. Curves showing the dependence of the notch toughness on temperature for material C.

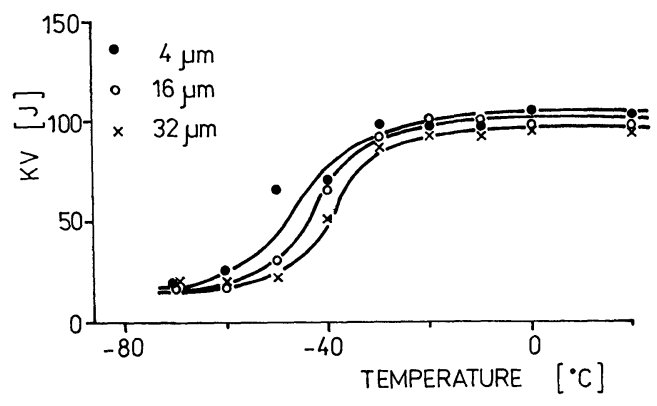

Fig. 9. Gurves showing the dependence of the notch toughness on temperature for material Al for various surface quality. 
various thicknesses of the individual sheets in a specimen and/or for various surface unevenness.

\section{Discussion}

In this work the energy required for fracture, referred to unit fracture area of a pack, is assumed to be the same as that referred to unit area of the individual sheet, i.e.

$$
\frac{U_{c}}{B_{c} h}=\frac{n U_{1}}{n B_{1} h}=\frac{U_{1}}{B_{1} h}=\mathrm{KCV}
$$

where, $U:$ the fracture energy

$B:$ thickness of specimen

$h$ : length of ligament

$n$ : the number of sheets in a pack.

The index $c$ refers to the total pack, while the index 1 to one sheet of the pack. In fact the specific energy of the sheets of a pack may be different due to various kinds of fractures (ductile fracture, cleavage fracture) and to a lesser extent due to the plastic strain in parallel with the thickness of the pack $B$. However, the latter effect is probably too low and is neglected in further considerations.

\section{Zone of Shear Fractures}

The maximum values of energy for fracture of the investigated specimens are summarized in Fig. 10. At first glance great differences are obvious in the specific-energy values of the three steel grades. These differences are diminishing with decreasing thickness of sheet $\left(B_{1}\right)$ and below a thickness of $1 \mathrm{~mm}$ these differences are approaching to values determined by the theoretical relationship (4). The dynamic yieldlimit values for a given loading rate for the examined materials are listed in Table 2. Figure 10 shows very good linkage of the theoretical functions (4) with the curves determined experimentally.

In the case of thicker individual sheets we come across considerable differences not only among the materials $\mathrm{A}$ to $\mathrm{C}$, but even among different sites of a single sheet (A1 and A2). Let us make an insight into these differences from the mechanical point of view. The primary metallurgical reasons resulting into the described results will be discussed later. On the basis of investigation into the fracture surface it

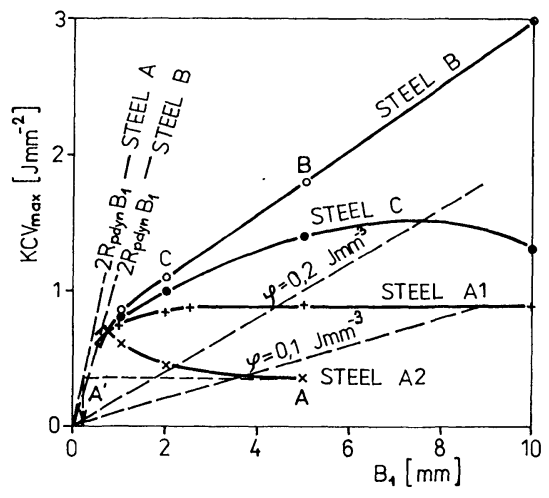

Fig. 10. Dependence of the energy of shearing fractures on the thickness of sheets. can be seen that the drop in the specific fracture energy is conditioned by two mechanisms, i.e., the lamellar cracking and the cleavage fracture.

Material A reveals considerable differences in the fracture stresses in longitudinal and transversal direction as it follows from Table 2. In this respect the longitudinal direction of the original sheet is in conformity with the direction of $\sigma_{1}$-stress and the transversal direction with $\sigma_{2}$-stress in the thin specimens, see Fig. 3(a). Thus, the direction of $\sigma_{3}$-stress is identical with that transversal to the front surfaces of the original sheet and one can expect that the fracture stress $R_{f 3}$ would be smaller or in the best case the same as the fracture stress of the transversal direction (Table 2). When drawing now the fracture stresses $R_{f 1}$ and $R_{f 3}$ into Fig. 3(b), it is obvious that at displacement of the Mohr's circle to the right-hand side due to the loading dynamics the stress $\sigma_{3}$ attains the relevant fracture stress earlier than $\sigma_{1}$-stress.

This condition can be mathematically expressed as follows ${ }^{9}$

$$
R_{f 1}-R_{f 3}>\sigma_{1}-\sigma_{3}
$$

Accordingly, the material will be torn in planes parallel with the front surfaces of sheets, see Photo. 2(a). Crack initiation is however associated with fundamental variations in the components of stress tensor that will be now similar to those in the pack of very thin sheets. The specific fracture energy is falling down to the level corresponding to that respective thickness. For example, point $\mathrm{A}$ in Fig. 10 lies in conformity with point $\mathrm{A}^{\prime}$ on the theoretical slope line $(d \mathrm{KGV} / d B)_{B=0}$ with a thickness $B_{1} \sim 0.3 \mathrm{~mm}$. Al-

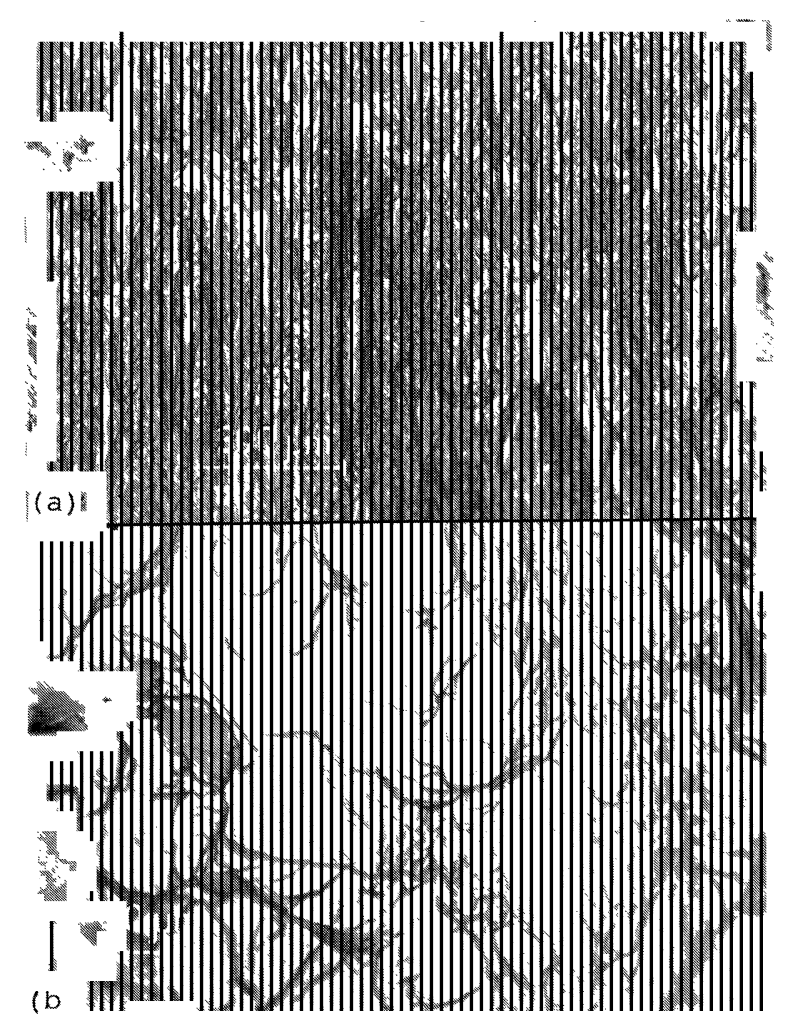

Photo. 2. Appearance of the fracture surfaces of material A. 
though that value corresponds in principle to the distances of the main cracks (see Photo. 2(a)), the equivalent mean distance of cracks will be somewhat longer as also the cleavage fracture has taken part at the decrease of the specific fracture energy of specimens from point A (Fig. 10). This can be seen in Photo. 2(b) showing, in addition to the ductile shear fracture, the facets of a cleavage fracture featured by less demands for energy.

The differences in the fracture-energy values of the various sites of A-sheet (Fig. 10) will be most likely due to different heat-level control in the two sites in the course of the final period of rolling as it follows for example from the comparison of the results of the D-T tests run with the same sheet in as-normalized and non-annealed state. ${ }^{10}$

The fracture energy of specimens prepared of Bmaterial is considerably greater than that of A-material. The fracture surfaces are characterized by ductile voids as it follows from Photo. 3(a) showing a photograph of the fracture surface of a specimen of Bpoint (Fig. 10) and in Photo. 3(b) showing the fracture of a single sheet in the point $\mathrm{C}$. In the case of thinner sheets illustrated in Photo. 3(b) $\left(B_{1}=2 \mathrm{~mm}\right)$ the individual voids are larger and more elongated than those of thicker sheets $\left(B_{1}=5 \mathrm{~mm}\right)$ shown in Photo. $3(\mathrm{a})$, which may be due to a smaller component of $\sigma_{3}$-stress at thinner sheets. ${ }^{11)}$ All the photographs from the scanning microscope are orientated so that the notch occurs towards the top part of picture.

Comparison of the temporal courses of the force during fracture for the points A, B and C of Fig. 10 is illustrated in Fig. 11(a). Here, the individual photographs from Polaroid camera are newly drawn

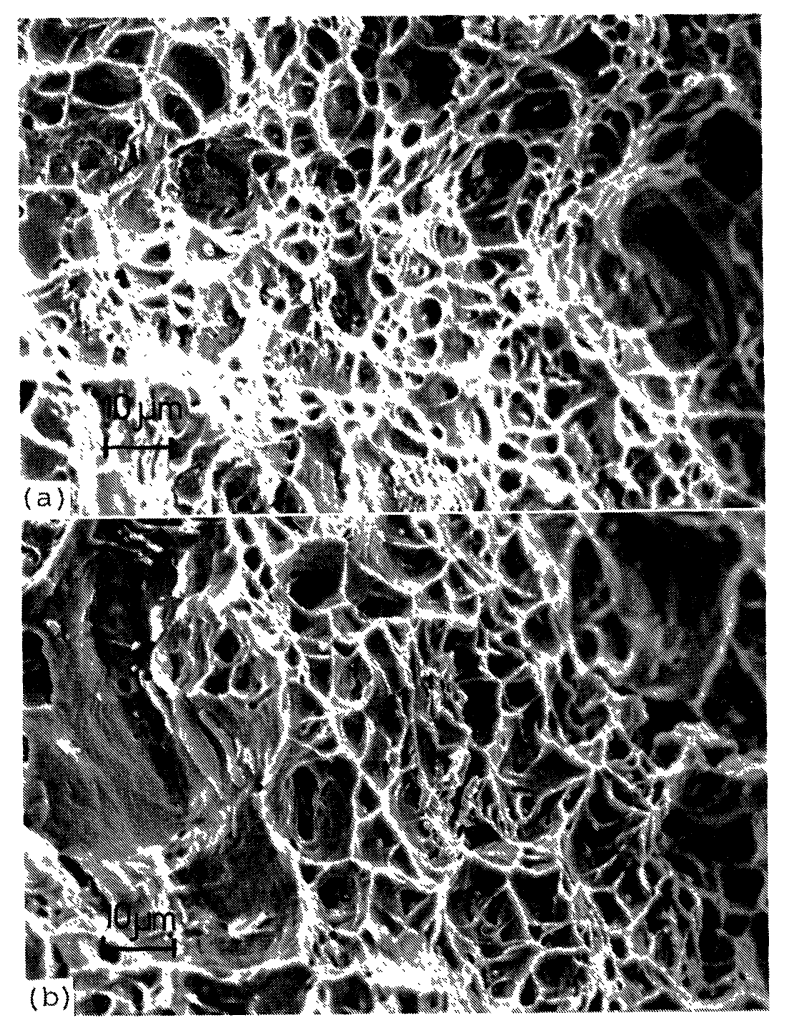

Photo. 3. Appearance of the fracture surfaces of material B. for the sake of lucidity in complex. It is obvious that the maximum forces do not differ much one from another (about $18 \%$ ). However, the energy required for crack origination is quite different and the highest energy occurs at specimens with components some $5 \mathrm{~mm}$ thick of point $\mathrm{B}$, lesser is at specimens of components $2 \mathrm{~mm}$ thick of point $\mathrm{C}$ and the least energy encounters at specimens of components $5 \mathrm{~mm}$ thick of point A. This sequence is also in agreement with the time of duration of the proper fracture process. The thinner the components, the shorter is the time required for fracture. This conclusion is in conformity with the time of duration of fractures of specimens $5 \mathrm{~mm}$ thick of point A, because the stress $\sigma_{3}$ tears them into parallel cracks with the front surfaces of sheets. The number of sheets in a pack with constant pack thickness affects the result negligibly. The maximum force required for crack origination in a sheet appears to be linearly summed-up for the number of $\underline{n}$ sheets. Figure 11(b) shows the course of forces redrawn from the photographs taken by Polaroid camera for specimens of point A (Fig. 10), whereby one specimen consisted of components $5 \mathrm{~mm}$ thick and the other of a single specimen $5 \mathrm{~mm}$ thick. The force required for crack origination in a composed specimen is here double that for a simple specimen.

The increasing amount of energy required for fracture of thick specimens made of B-material is intimately associated with a more complex configuration of the fracture surfaces as dealt with in detail in the second part of this article.

The concept of volume shearing fracture energy $\varphi$ [Eq. (2)] is illustrated in Fig. 10 for two values, i.e., $\varphi=0.1$ and $\varphi=0.2 \mathrm{Jmm}^{-3}$. It is evident that for specimens of a greater thickness the parameter $\varphi$ is in fact a formal concept. It has physical significance for small thicknesses $B_{1}$ only at which the shear fracture occurs and the stress $\sigma_{3}$ perpendicular to the front surfaces is small.

Figure 9 shows the dependences of $\mathrm{KCV}(\mathrm{t})$ for specimens composed of five components with milled surface to obtain various surface unevenness. The fracture behaviour is likely to be influenced by stress due to machining. However the differences are
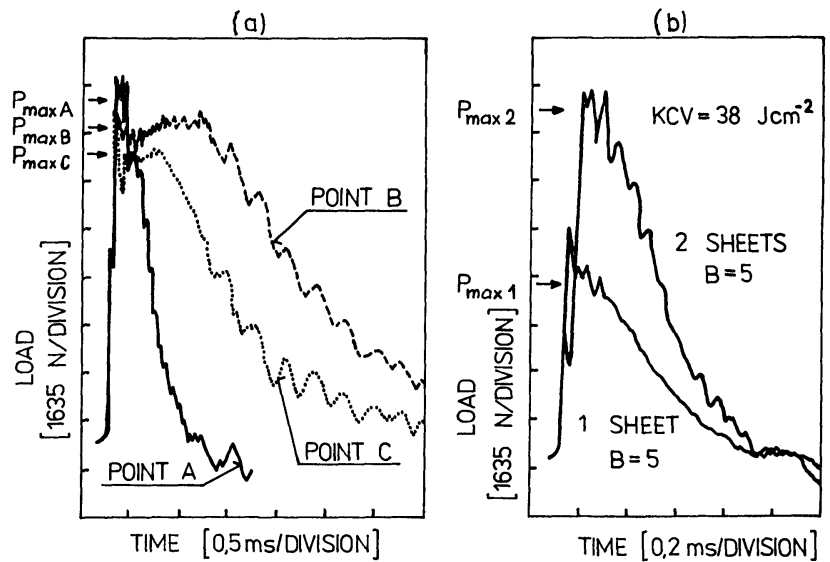

Fig. 11. The course of forces in dependence on time during fracture. 
small. The transient curves are displaced towards higher temperatures.

\section{Zone of Mixed and Brittle Fractures}

The previous chapter has dealt with the decrease in the fracture energy in association with diminishing thickness of the individual components of a pack. In the case of very thin sheets failured by pure shear that energy is very low and thus, these fractures are dealt with as "ductile cleavage ".11)

It is a well-known fact that a drop in temperature has substantial effect on decrease of the fracture energy of steel pieces. However, the thinner the specimen, the more displaced is its transient temperature to lower temperatures. The energy required for a ductile fracture remains approximately constant up to the field of low temperatures as can be seen from Figs. 5 to 8 . Then, the drop in energy in the transient part of curve is caused either by a mixed fracture of all the individual components of the pack or by a ductile fracture of several components and by cleavage fracture of the residual components. The latter encountered for example with the specimen of point A (Fig. 7), where two out of five sheets have broken by the cleavage fracture and the other three sheets by the ductile fracture.

The sheets $5 \mathrm{~mm}$ thick (point B in Fig. 7) failured at the same temperature of $-60{ }^{\circ} \mathrm{C}$ reveal already completely the cleavage fracture. At temperatures lower than $t_{p}$ of the composed specimens all the individual sheets have broken by cleavage fracture.

When plotting the temperatures $t_{p}$ of the transient curves in a relationship with thickness $B_{1}$, one obtains diagram shown in Fig. 12. As can be seen each of the materials reveals somewhat different course of $t_{p}\left(B_{1}\right)$ function.

Nevertheless, this relationship has informative character down to temperatures at which the measurements were run, i.e., down to $-200{ }^{\circ} \mathrm{C}$. As it follows from Fig. 12 the segment from -200 to -273 ${ }^{\circ} \mathrm{C}$ is small in log-coordinates, however, it is important from the physical point of view and thus, the results cannot be extrapolated.

Figure 12 provides information about one of the parameters characterizing the transient curves illustrated in Figs. 5 to 8 . In fact the resistance to dynamic bending of material is function of $\mathrm{KGV}_{\max }$,

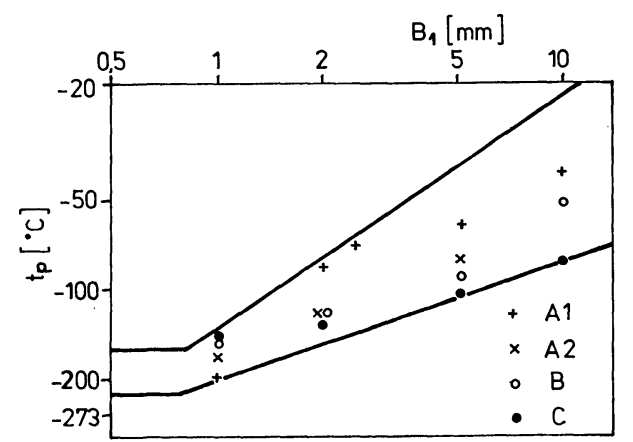

Fig. 12. Dependence of the threshold temperature of the transient curve on the sheet thickness.
$\mathrm{KGV}_{\min }, t_{p}, \Delta t$ (which is the temperature difference referring to a change of the specific energy $\mathrm{KCV}_{\max }$ into $\left.\mathrm{KGV}_{\min }\right), B_{1}$ and $R_{p \text { dyn }}$. These parameters allow us to define the dimensionless factor $z$ as follows

$$
Z=\frac{\mathrm{KGV}_{\max }-\mathrm{KGV}_{\min }}{R_{p \text { dyn }} \cdot B_{1}} \cdot \frac{\left|t_{p}\right|}{\Delta t}
$$

where, $\left|t_{p}\right|$ : the absolute value of $t_{p}$-temperature.

Equation (11) was expressed in numbers for the results given in Figs. 5 to 8 and, in dependence on $B_{1}$, it is plotted in diagram shown in Fig. 13. As it follows here the results for each of the examined quality fall on a curve with a relatively small scatter. The factor $\underline{Z}$ is differentiating quite good the various grades of thin sheets. The differences between the individual steel grades are decreasing with diminishing thickness. The linear relationship in log-log coordinates induces to extrapolation of $z$-figures for small thicknesses $B_{1}$ out of the measured range. Nevertheless, from Eq. (11) it can be found that with diminishing thickness one obtains

$$
\begin{aligned}
\lim _{B_{1} \rightarrow 0} Z & =\frac{\mathrm{KVG}_{\max }}{R_{p \mathrm{dyn}} B_{1}} \cdot\left(1-\frac{\mathrm{KGV}_{\min }}{\mathrm{KGV}_{\max }}\right) \cdot \frac{\left|t_{p}\right|}{\Delta t} \\
& =2\left(1-\frac{\mathrm{KGV}_{\min }}{\mathrm{KGV}_{\max }}\right) \cdot \frac{\left|t_{p}\right|}{\Delta t} \rightarrow 0 \ldots \ldots . .
\end{aligned}
$$

as the quotient of $\mathrm{KCV}_{\min }: \mathrm{KCV}_{\max }$ converges to unit with diminishing thickness $B_{1}$ and $\mathrm{KCV}_{\max } \rightarrow$ $2 R_{p \mathrm{dyn}} B_{1}$ by Eq. (4).

Thus, by extrapolation of the straight lines of Fig. 13 one would obtain erroneous results. In the range of $B_{1}$ of Fig. 13 the individual courses can be expressed by the following polytropic function

$$
Z B_{1^{\mathrm{s}}}^{k_{5}}=k_{6}
$$

whereby the constants $k_{5}$ and $k_{6}$ of the examined steels are given in Table 3. As can be seen the steels B and $\mathrm{C}$ have exponent $k_{5}$ approaching to unit, while with impairing resistance to dynamic bending of Asteel the exponent $k_{5}$ is rising and the constant $k_{6}$ is decreasing.

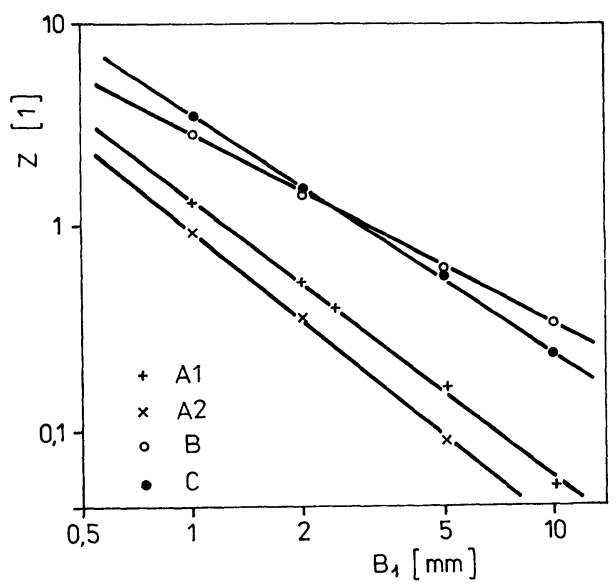

Fig. 13. Dependence of $Z$-parameter on the thickness of the individual sheets. 
Table 3. The constants $k_{5}$ and $k_{6}$ for our testing.

\begin{tabular}{|c|c|c|c|c|}
\hline \multirow{2}{*}{$\begin{array}{c}\text { Constant } \\
\text { of } \\
\text { materials }\end{array}$} & \multicolumn{4}{|c|}{ Material } \\
\hline & Al & $\mathrm{A} 2$ & B & $\mathrm{C}$ \\
\hline$k_{5}$ & 1.361 & 1.467 & 0.928 & 1.169 \\
\hline$k_{6}$ & 1.353 & 0.968 & 2.805 & 3.457 \\
\hline
\end{tabular}

\section{Conclusion}

In the article we have dealt with the ductile and brittle fracture of specimens composed of thin sheets in shape of Charpy V. The theoretical analysis into the dependence of the "crack driving force" $G_{c}$ or the specific energy $\mathrm{KCV}$ on the sheet thickness $B_{1}$ shows that the function $G_{c}\left(B_{1}\right)$ can be split into four zones characterized by different course of fracture. In the first zone it is the s.-c. "ductile cleavage" in which the relationship $G_{c}\left(B_{1}\right)$ is linear. The second zone is characterized by origination of complex configuration of the fracture surfaces of comparable thickness, which results in fractures well-demanding from the viewpoint of energy. In the third zone the mixed fracture originates. That part of curve $G_{c}\left(B_{1}\right)$ is originating in principle by the transient curves $G_{c}(t)$ of specimens of a greater thickness. The fourth zone is characterized by occurrence of brittle fractures only.

The experimental part of the work underlines the effect of different material properties in the longitudinal and in transversal direction on the specific energy of the shear fractures.

The analysis of the temporal course of forces at failure of the composed specimens shows that

(1) the maximum force for fracture of a pack is given by the algebraic sum of forces required for fracture of the individual sheets providing that the kind of fracture (ductile or cleavage) is the same for all sheets,
(2) the energy required for fracture and the duration of the fracture process are decreasing with the thickness of the individual components of a pack. It was found that the parameter

$$
Z=\frac{\mathrm{KGV}_{\max }-\mathrm{KGV}_{\min }}{R_{p \mathrm{dyn}} \cdot B_{1}} \cdot \frac{\left|t_{p}\right|}{\Delta t}
$$

refers reasonably to the resistance of the studied set of specimens to fracture at dynamic bending. For the sake of comparison of the specific fracture energy of thin sheets with the standard Charpy $\mathrm{V}$ specimens all the parts of the specimens were prepared by milling of heavy plates. Therefore, the study does not include the effect of the surface strengthening of sheets by rolling.

\section{REFERENCES}

1) W. A. Poynton and J. R. Christian: Proceedings " Crack Propagation in Pipe lines ", The British Gas Corp., Newcastle Upon Tyne, 1974, Paper No. 5.

2) E. A. Lange: Proceedings " Toward Improved Ductility and Toughness ", Climax Molybdenum Co., Tokyo, (1971), 33.

3) R. W. Judy, Jr. and R. J. Goode: ASME Spec. Tech. Publ., 536 (1973), 377.

4) G. T. Hahn, R. G. Hoagland and A. R. Rosenfield: Proceedings "Dynamic Fracture Toughness", The Welding Inst. \& ASM, London, (1976), 237.

5) J. F. Knott: Fundamentals of Fracture Mechanics, Butterworths Sci. Publ., London, (1961), 41 \& 119.

6) J. Němec: Toughness and Strength of Steel Parts, Czechoslovak Academy of Sciences, Prague, (1961), 142.

7) E. A. Lange: ASTM Spec. Tech. Publ., 466 (1970), 241.

8) N. Taniguchi: Proceedings "Fracture Mechanics and Technology", II, Univ. of Hong Kong \& Lehigh Univ., Hong Kong, (1977), 1397.

9) G. Baldi and G. Buzzichelli: Metal Sci., (1978), Oct., 459.

10) J. Kucěra: not published yet.

11) J. Koutský, F. Jandoš and V. Karel: Fractures in Steel Parts, SNTL, Prague, (1976), 81. 